\title{
Pollution De L'Air Interieur Et Comportements Des Asthmatiques Lies A La Gestion De La Maladie Dans Les Menages De La Commune De Yopougon (Côte D’ivoire)
}

\author{
Kouadio Ahou Rosine \\ UFR Sciences Médicales Abidjan, Université Félix Houphouët-Boigny de \\ Cocody, Côte d'Ivoire \\ Adje N'goran Pascal \\ UFR Sciences de l'Homme et de la Société, Département de Sociologie, \\ Université Félix Houphouët-Boigny de Cocody, Côte d'Ivoire \\ Yao Kouakou Daniel \\ UFR Sciences Sociale et Humaine, Université Jean Lorougnon Guédé de \\ Daloa, Côte d'Ivoire

\section{Ouattara Soualiho} \\ UFR Sciences Médicales, Département de physiologie, Université Félix \\ Houphouët-Boigny de Cocody, Cote d’Ivoire
}

\section{Doi:10.19044/esj.2021.v17n29p74}

Submitted: 17 June 2021

Accepted: 26 July 2021

Published: 31 August 2021
Copyright 2021 Author(s)

Under Creative Commons BY-NC-ND 4.0 OPEN ACCESS

Cite As:

Rosine K. A., Pascal A. N., Daniel Y. K., \& Soualiho O. (2021). Pollution De L'air Interieur Et Comportements Des Asthmatiques Lies A La Gestion De La Maladie Dans Les Menages De La Commune De Yopougon (Côte D’ivoire). European Scientific Journal, ESJ, 17(29), 74. https://doi.org/10.19044/esj.2021.v17n29p74

\section{Résumé}

La prévalence de l'asthme est en constante augmentation et les facteurs environnementaux dont la pollution de l'air intérieur sont incriminés.

La présente étude, réalisée dans la commune de Yopougon en Côte d'Ivoire, vise à analyser le comportement des asthmatiques dans la gestion de la maladie en relation avec la pollution de l'air intérieur. Sur cette base, la théorie du choix rationnel a été retenue pour étayer les comportements des asthmatiques interviewés. Une méthode mixte a été adoptée avec la conduite d'entretiens semi dirigés individuels auprès de 31 patients asthmatiques. L'enquête quantitative a concerné 200 patients asthmatiques.

Les résultats indiquent que $63 \%$ des enquêtés combinaient l'utilisation du charbon de bois et celle du gaz butane domestique. Les différents 
combustibles, gaz butane, charbon de bois et bois de chauffe génèrent des polluants lors de leur utilisation; mais, le gaz butane est celui qui pollue le moins. De même, 68\% des asthmatiques optent pour un traitement mixte (moderne et traditionnel). L'asthme dans ce contexte n'est pas contrôlé, 41\% ont un asthme persistant, léger et font environ trois crises par semaine.

Mots clés: Asthme, Comportement, Pollution De L’Air Intérieur, Combustibles, Traitement

\title{
Indoor Air Pollution And Asthma Patients’ Behaviour In Controlling The Disease In The Households Of The Municipality Of Yopougon (Côte D’ivoire)
}

\author{
Kouadio Ahou Rosine \\ UFR Sciences Médicales Abidjan, Université Félix Houphouët-Boigny de \\ Cocody, Côte d'Ivoire \\ Adje N'goran Pascal
}

UFR Sciences de l'Homme et de la Société, Département de Sociologie, Université Félix Houphouët-Boigny de Cocody, Côte d'Ivoire

\section{Yao Kouakou Daniel}

UFR Sciences Sociale et Humaine, Université Jean Lorougnon Guédé de Daloa, Côte d'Ivoire

\section{Ouattara Soualiho}

UFR Sciences Médicales, Département de physiologie, Université Félix Houphouët-Boigny de Cocody, Cote d’Ivoire

\begin{abstract}
The prevalence of asthma is constantly increasing and environmental factors including indoor air pollution are blamed.

This study, which took place in the commune of Yopougon, aims to analyze asthma sufferers' behaviour in managing the disease in the face of indoor air pollution. To this end, the theory of rational choice was convoked to shed light on the behaviour of asthma patients who participated in the study. A mixed method was used with the conduct of semi-structured individual interviews with 31 asthma patients. Quantitative data were collected from 200 other asthma patients.

Results reveal that $63 \%$ of the respondents combined the use of charcoal and that of domestic butane gas. The miscellaneous fuels: butane gas, charcoal and firewood generate pollutants during their use; but, butane gas is the one that pollutes the least. In addition, $68 \%$ of asthma patients opt for mixed treatment (modern and traditional). Under these circumstances, asthma
\end{abstract}


is not controlled, as $41 \%$ of the participants have persistent, mild asthma and undergo about three attacks per week.

Keywords: Asthma, Behavior, Indoor Air Pollution, Fuels, Treatment

\section{Introduction}

L'asthme est une maladie non transmissible de première importance qui, selon les statistiques de l'OMS (2017), touchait plus de 339 millions de personnes dans le monde en 2016. C’est une maladie chronique caractérisée par l'inflammation des voies aériennes et une difficulté respiratoire (dyspnée) sifflante. Ce dysfonctionnement est lié à une réactivité anormale des voies aériennes dont le diamètre diminue exagérément en réponse à différents facteurs déclencheurs (Puddu et al ; 2003). Parmi ces facteurs déclencheurs figurent ceux liés à l'environnement.

Les facteurs environnementaux, responsables du développement, du déclenchement et de l'aggravation ou de l'exacerbation de l'asthme chez les sujets prédisposés, renvoient aux allergènes de l'habitat (acariens, animaux domestiques...), la pollution de l'air (à l'intérieur et à l'extérieur des locaux).

La pollution de l'air, selon l'OMS ${ }^{1}$, est «la contamination de l'environnement intérieur ou extérieur par un agent chimique, physique ou biologique qui modifie les caractéristiques naturelles de l'atmosphère ». Les polluants les plus rencontrés dans ces cas sont la fumée, la moisissure, les allergènes, le monoxyde de carbone (CO), le dioxyde de carbone $\left(\mathrm{CO}_{2}\right)$, l'oxyde d'azote $\left(\mathrm{NO}_{\mathrm{x}}\right)$, l'ozone $\left(\mathrm{O}_{3}\right)$, les composés organiques volatils (COV), les particules fines $\left(\mathrm{PM}_{10}\right.$ et $\left.\mathrm{PM}_{2,5}\right)$, etc. (Billionnet, 2012). Ces polluants peuvent également être d'origine naturelle (éruption volcanique) ou anthropique (diverses activités humaines).

Pour ce qui est de la pollution de l'air intérieur, les sources potentielles sont entre autres les animaux domestiques, la moisissure, les matériaux de construction et d'équipement, les produits de nettoyage, la fumée de tabac, l'utilisation de combustibles solides pour la cuisson et le chauffage, l'introduction de l'air extérieur à l'intérieur des endroits clos (habitations, lieux de travail), etc. (Billionnet, 2012).

Plusieurs activités humaines à l'intérieur des logements, en effet, produisent des substances et des particules susceptibles de provoquer des crises d'asthme. C'est le cas des Composés Organiques Volatils (COV) ${ }^{2}$, issus

\footnotetext{
${ }^{1}$ Site de l’OMS (consulté en septembre 2019)

https://www.who.int/topics/air_pollution/fr/\#: :text=Par\%20pollution\%20de\%20l'air,caract\%C3\%A9r istiques\%20naturelles\%20de\%20l'atmosph\%C3\%A8re.

${ }^{2}$ Une cinquantaine de COV sont communément retrouvés dans l'environnement intérieur. Par définition ils sont volatils à température ambiante (Pauli, de Blay, Krieger et Bessot, 2016). Ils appartiennent à 9 groupes de composés chimiques: alcanes, cycloalcanes et alkènes, hydrocarbures aromatiques ou benzéniques, hydrocarbures halogénés, terpènes, aldéhydes, cétones, alcool, esters.
} 
des produits de construction, de décoration, d'ameublement, d'entretien et de bricolage et les activités humaines liées aux besoins essentiels (cuisine, hygiène) ou autres tels que le tabagisme, l'utilisation de bougies et d'encens (Billionnet, 2012., Nicolas et al; 2013).

Par ailleurs, l'utilisation de combustibles solides comme la bouse de vache, le bois, les résidus agricoles ou le charbon de bois produisent du monoxyde de carbone (CO), des particules fines $\left(\mathrm{PM}_{2,5}\right.$ et $\left.\mathrm{PM}_{10}\right)$, des hydrocarbures et de l'oxyde d'azote (NO) dans l'air intérieur (Lang, 2006). Il convient de préciser que toutes ces substances issues de l'utilisation de combustibles solides sont des polluants.

De même, la présence d'humidité dans un espace clos favorise l'apparition de moisissures (Dallongeville, 2015). Cette humidité peut avoir différentes sources en lien avec la structure du bâtiment (mauvaise isolation, fuite, circulation d'air insuffisante et inadaptée) et les activités humaines (cuisine, ménage). Ces différentes activités humaines à l'intérieur du logement ont un impact négatif sur la qualité de l'air dans les domiciles et cela constitue une menace pour la santé de leurs résidents.

Toutes ces sources de pollution de l'air intérieur représentent une menace pour la santé humaine en générale mais plus particulièrement pour les personnes vivant avec des maladies respiratoires comme l'asthme (Liu et al, 2018). Cependant, l'on observe que malgré ces risques liés à leur état de santé, les asthmatiques ne prennent pas suffisamment de précautions pour une amélioration de leur santé. En Côte d’Ivoire, les données sur la prévalence de l'asthme sont encore parcellaires. Dans la ville de Bouaké, cette prévalence est de 11,3\% chez les adolescents en milieu scolaire (Koné et al, 2018). La ville de Korhogo quant à elle enregistre une prévalence de 5,4\% chez les adolescents en milieu scolaire (Koné et al, 2018). Dans ce contexte, la présente recherche vise à analyser le comportement des patients asthmatiques dans la gestion de la maladie relativement à la pollution de l'air intérieur. De manière spécifique, il s'agit de décrire le cadre de vie des asthmatiques et les types de combustibles utilisés, de montrer les effets induits sur leur état de santé à travers la fréquence des crises, le mode de vie des patients asthmatiques et leurs choix thérapeutiques.

Pour y parvenir, la théorie utilisée dans ce travail est celle du choix rationnel. Cette théorie est un modèle d'explication pour interpréter les pratiques et comportements des individus (Bozonnet, 2007). La théorie du choix rationnel dans ce travail est nécessaire pour comprendre les motivations, le sens cognitif et interprétatif dans le comportement des asthmatiques dans la gestion de la maladie. 


\section{Methode}

La présente étude a été menée dans la commune de Yopougon (Côte d'Ivoire), qui est l'une des treize communes d'Abidjan, la capitale économique du pays. Située dans la zone ouest d’Abidjan, un peu excentrée, elle se trouve entre la forêt du banco et la lagune Ebrié. Sa superficie est de 153,06 km² avec une population d'environ 1071543 d'habitants selon les résultats de l'Institut National de Statistiques (INS, 2014).

Diverses raisons fondamentales ont guidé le choix de la commune de Yopougon. Il s’agit du projet de la Chaire écosanté en pollution de l'air intitulé ChairePol qui avait ciblé cet espace communal. En plus, Yopougon est non seulement la plus vaste commune d'Abidjan avec un trafic routier dense, mais, également elle abrite la plus grande zone industrielle du pays. En outre, le développement des activités informelles telles que le site de fumage de poisson et de viande utilisant les feux domestiques prend de l'ampleur. Ce site est logé dans le marché du quartier de Niangon nord communément appelé « Lubafrique » (Yopougon info, 2014). Ces caractéristiques sont susceptibles de générer une pollution de l'air dans la commune.

Figure 1. Cadre de l'étude

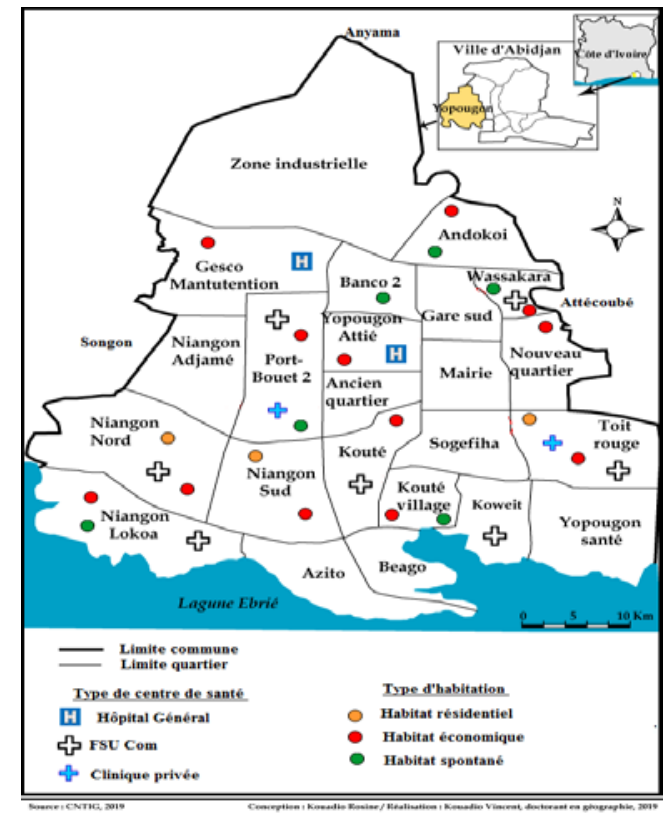

La figure 1 présente les centres de santé classés en trois catégories : les Hôpitaux Généraux (HG), les Formations Sanitaires Urbaines et Communautaires (FSU Com) et les Cliniques privées. De même, cette figure présente les habitations sous trois types: les habitats résidentiels, économiques et spontanés (précaires). 
L'échantillon de l'étude porte sur la population asthmatique de la zone communale. Avec l'échantillonnage de type accidentel, 87 patients asthmatiques ont été recensé avec l'appui du corps médical dont les médecins. Ces personnes ont été rencontrées dans différents centres de santé de ladite commune. Les centres de santé concernés sont : le Centre Hospitalier et Universitaire (CHU) de Yopougon, l'Hôpital Général Attié, les Formations Sanitaires Urbaines (FSU) des quartiers Wassakara, Toit-Rouge, Port-Bouët 2, Lokoa et la Clinique privée le Grand Centre. Dans ces différents centres, seuls le CHU et la clinique le Grand Centre disposent de Pneumologue en leur sein. Ces deux centres sont les plus fréquentés par les enquêtés.

Tableau 1. Répartition des enquêtés selon les centres de santé

\begin{tabular}{|c|c|c|}
\hline $\mathbf{N}^{\circ}$ & Centre de santé & Effectif \\
\hline 1 & Centre Hospitalier et Universitaire (CHU) & 46 \\
\hline 2 & Hôpital Général Attié & 05 \\
\hline 3 & Formation Sanitaire Urbaine de Wassakara & 03 \\
\hline 4 & Formation Sanitaire Urbaine de Toit-Rouge & 12 \\
\hline 5 & Formation Sanitaire Urbaine de Port-Bouët 2 & 02 \\
\hline 6 & Formation Sanitaire Urbaine de Lokoa & 01 \\
\hline 7 & Clinique privée le Grand Centre & 18 \\
\hline \multicolumn{2}{|c|}{ Total } & $\mathbf{8 7}$ \\
\hline
\end{tabular}

Source: Données de l'étude

Avec ces (87) asthmatiques obtenus dans les centres de santé, la technique d'échantillonnage de type boule de neige a été utilisée pour atteindre (231) personnes souffrant d'asthme dans la commune de Yopougon. Il faut dire que ces asthmatiques rencontrés hors des centres de santé présentaient leurs bulletins de consultation et ordonnances, accompagnés de bulletins d'examens. Certains parmi eux ont pu effectuer ces examens et d'autres non. Dans cet échantillon, le sexe masculin était majoritairement représenté, avec $52,5 \%$ contre $47,5 \%$ de femmes. La moyenne d'âge était de 32 ans. Ces enquêtés étaient essentiellement des fonctionnaires (33,5\%), des agents du secteur privé (32,5\%) et des élèves (18\%). Les ménagères combinées aux sans emploi représentent $8,5 \%$ et les étudiants ont une proportion de $7,5 \%$. Le niveau d'étude majoritairement représenté était le niveau secondaire avec 90 individus, soit $45 \%$ des asthmatiques.

Une approche mixte a été adoptée dans la réalisation de cette recherche.

En ce qui concerne l'approche qualitative, l'échantillonnage à choix raisonné a été utilisé. L'échantillon constitué de 31 asthmatiques a été sélectionné sur la base de la disponibilité des enquêtés, de la durée de la maladie dans leur vie (cinq ans au moins), de leur résidence à Yopougon depuis au moins un an. Tous les âges étaient concernés par cette étude. Cependant, les personnes de moins de 18 ans étaient assistées par l'un des 
parents. Une fiche de consentement éclairé était signée par l'enquêté et une fiche d'information leur était adressée avant le démarrage de l'entretien à leur domicile. Les entretiens étaient orientés sur leurs connaissances de l'asthme, de la pollution de l'air intérieur et des différentes pratiques sociales dans le ménage qui peuvent améliorer ou dégrader leur état de santé.

L'approche quantitative s'est quant à elle déroulée avec les 200 autres asthmatiques obtenus par la technique d'échantillonnage accidentel et boule de neige. Le questionnaire a été administré aux patients dans leur domicile respectif sous présentation de la fiche de consentement éclairé, signée par l'enquêté et la fiche d'information. Les questions ont ainsi porté sur quatre thèmes essentiels : les caractéristiques sociodémographiques, leurs connaissances de l'asthme et de la pollution de l'air intérieur, les pratiques adoptées dans la gestion de la maladie et la fréquence des crises d'asthme.

Les entretiens retranscrits ont fait l'objet d'une analyse de contenu qualitatif de type thématique. Cette approche a permis de faire ressortir l'intérêt particulier accordé aux thèmes, aux mots ou aux concepts employés par les enquêtés (Abric, 2005). Le questionnaire a fait l'objet d'une analyse statistique et spécifiquement de statistiques descriptives à l'aide des logiciels Sphinx et Excel. Les résultats obtenus ont permis de concevoir des tableaux de distributions des fréquences et des représentations graphiques des données (N’Da, 2002).

Malgré les dispositions mises en place, dans le cadre de la réalisation de cette recherche, notre enquête ne s'est pas déroulée sans difficultés. La principale difficulté à laquelle nous avons été confrontés fut l'indisponibilité de certains asthmatiques. Des rendez-vous pris au téléphone n'ont pu être honorés par certains asthmatiques en raison d'un imprévu ou de l'oubli. Ces rendez-vous ont donc dû être reprogrammés. Des enquêtés étaient également retissants.

\section{Resultats}

\section{Description des habitations des asthmatiques}

Tableau 1. répartition des enquêtés selon le type d'habitat de février à juillet 2018

\begin{tabular}{|c|c|c|}
\hline Type d'habitat & Effectif & Pourcentage \\
\hline Spontanés & 47 & $24 \%$ \\
\hline Economiques & 143 & $71 \%$ \\
\hline Résidentiel & 10 & $5 \%$ \\
\hline Total & 200 & $100 \%$ \\
\hline
\end{tabular}

Il ressort de ce tableau que les personnes rencontrées au cours de l'enquête vivent dans divers types d'habitats : précaires ou spontanés, économiques et hauts standings ou résidentiels.

Trois caractéristiques du cadre de vie des asthmatiques se dégagent. 
La première est relative à l'existence de domiciles qui disposent de vastes cours avec des arbres et fleurs, de grandes fenêtres ouvertes témoignant d'une aération suffisante. Ces habitations sont généralement un peu excentrées et situées loin des grandes voies publiques à dense trafic routier.

Ensuite, l'on note la présence d'habitations situées en plein centre-ville avec un trafic dense, des maisons avec moins d'espace où l'aération des pièces (chambres, salons, cuisines) n'est pas suffisante et parfois mal entretenues (propreté de la maison, entretien du bâtiment, présence de moisissure par endroit). Certaines de ces maisons sont dotées de climatisation. Dans ce contexte, les habitants n'ont pas pour habitude d'ouvrir les fenêtres de peur que la poussière, les gaz d'échappement et les insectes n'entrent dans leurs maisons. Elles ne perçoivent pas l'ouverture des fenêtres comme une manière d'améliorer la qualité de l'air dans leurs domiciles.

Enfin, dans certains quartier "précaires ", la majorité des maisons est de type cour commune avec une aération insuffisante et très peu d'espace. Pour quelques ménages, des cuisines étaient faites en bois à proximité de la maison.

Les types d'habitations des enquêtés étant décrits, procédons à l'identification de leurs habitudes ou pratiques quotidiennes au sein de leurs ménages.

Au nombre des combustibles utilisés, nous distinguons : le gaz butane domestique, le charbon de bois et le bois de chauffe.

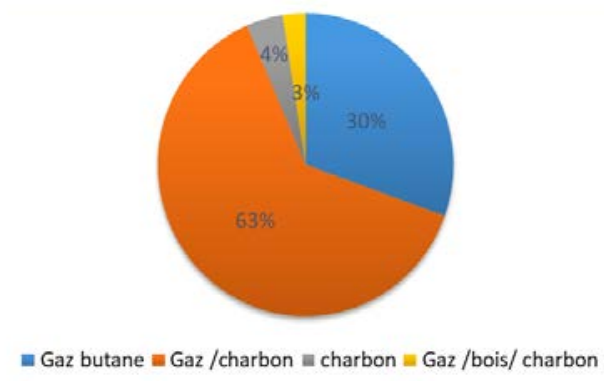

Figure 2. répartition de l'échantillon en fonction des combustibles utilisés dans les ménages de février à juillet 2018

La figure 2 montre que le combustible le plus utilisé est le couple gaz/charbon avec une proportion de $63 \%$ soit 126 patients asthmatiques de l'échantillon. Ceux utilisant exclusivement le gaz butane ou le charbon représentent respectivement $30 \%$ soit un effectif de 60 patients asthmatiques et $4 \%$ pour un effectif de 8 patients asthmatiques. Quant à la combinaison gaz/charbon/bois, elle est utilisée seulement par 6 patients asthmatiques soit $3 \%$ de la population de l'étude. Cette association de combustible s'explique par la diversité des éléments à faire cuire. 
L'utilisation du gaz et du charbon, indique que $45 \%$ des patients asthmatiques priorisent le charbon contre $55 \%$ qui utilisent le plus souvent le gaz, soit une différence de $5 \%$.

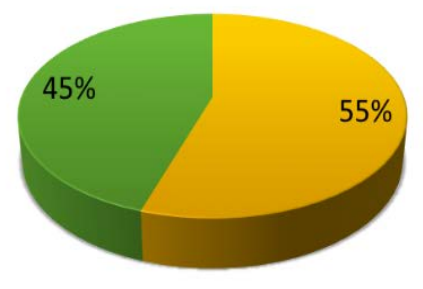

$\square$ Gaz+ $\square$ charbon +

Figure 3. répartition des enquêtés en fonction de l’utilisation combinée gaz/charbon dans les ménages de février à juillet 2018

Les extraits des entretiens font ressortir dans le cadre de la gestion de la maladie, un changement de comportement dans les habitations. Il s'agit notamment de la propreté des maisons et du rangement à l'intérieur des chambres.

« Mon fils faisait beaucoup de crises, ses crises étaient fréquentes. A chaque fois on va à l'hôpital, le médecin me pose plusieurs questions pour savoir comment la maison est, pour voir ce qui est à la base des crises. Je veille en tout cas sur la propreté de la maison mais, je n'avais pas pensé aux habits sales entassés dans un panier dans la chambre. En tout cas, je ne sais pas si c'est coïncidence mais depuis que ce problème a été réglé, j’ai déplacé ce panier, il y a du mieux ». (Femme adulte, 43 ans, Lubafrique)

« Le jour où je suis à la maison lors du nettoyage, elle [servante] essuie au lieu de balayer pour ne pas soulever la poussière en ma présence, étant donné que c'est dans la maison, la poussière ne se dissipe pas rapidement » (Femme adulte, 48 ans, Wassakara)

A travers ces propos, il apparaît que ces changements de comportements ou d'habitudes interviennent à la suite d'un constat effectué par le malade lui-même ou par son entourage dans le but d'une amélioration de l'état de santé de la personne asthmatique.

En plus, l'analyse du discours des patients asthmatiques révèle des pratiques antérieures à la maladie qui favorisent toutefois la bonne gestion de l'asthme.

"Quand je me lève chaque matin avant de faire le nettoyage de la maison, j'ouvre portes et fenêtres. La fenêtre de ma chambre même je ne la ferme jamais à moins que je voyage. J'ai toujours été comme ça bien avant que cette maladie ne se déclenche. C'est une habitude depuis petite que j'ai acquise avec mes parents » (femme adulte, 39 ans, quartier Lubafrique) . 
Notons que la distribution spatiale des habitations en milieu urbain avec la densité du trafic routier et l'absence de bitume contribuent largement à la gestion de l'asthme.

« Notre maison est située en bordure de cette rue non bitumée. C’est une rue fréquentée par les véhicules. Donc, la poussière ne finit pas ici. La situation de la maison nous fatigue » (Jeune homme, 27 ans, Sideci)

Le suivi du traitement de l'asthme selon les prescriptions médicales favorise la gestion de la maladie. Il atteste d'un changement de comportement du patient. Ainsi, ces propos extraits de nos entretiens relèvent :

«Mon fils a 14 ans et je lui explique comment prendre ses médicaments, mais si je ne fais pas la police derrière lui, ce n'est pas la peine. Je suis obligé de contrôler sinon il ne les prendra pas de lui-même. J'ai constaté que les moments de relâchements, quand je ne le suis pas correctement, les crises se répètent ». (Femme, 42 ans, Siporex)

\section{Fréquence des crises d'asthme}

L'asthme est une maladie qui se caractérise par des crises. La fréquence des crises chez la personne asthmatique concerne le nombre de fois que l'asthmatique fait la crise sur une période donnée. Dans ce travail, la classification suit les recommandations du Global Initiative for Asthma (GINA, 2019). L'évaluation de la fréquence des crises s'est faite par le nombre de crises survenues dans la semaine. Ce délai permettait à la personne asthmatique de pouvoir plus facilement se rappeler.
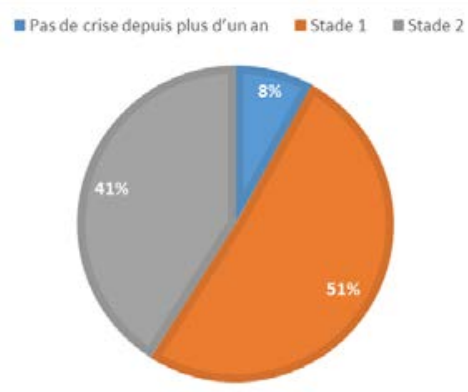

Figure 4. Répartition des enquêtés selon la fréquence des crises d'asthme (classification GINA, 2019) de février à juillet 2018

La figure ci-dessus indique que plus de la moitié des patients asthmatiques ont fait au moins une crise d'asthme par semaine soit une proportion de 51\%. Ils vivent un type d'asthme intermittent. Toutefois, $41 \%$ des patients asthmatiques vivent avec un asthme persistant léger, faisant ainsi, plus d'une crise par semaine. Depuis plus d'un an, 9\% des patients asthmatiques ne font plus de crises d'asthme. 


\section{Mise en relation du type de combustible et de la fréquence de crises}

Tableau 2. Analyse de la fréquence des crises par rapport aux combustibles de février à juillet 2018

\begin{tabular}{|c|c|c|c|c|c|c|}
\hline & \multicolumn{4}{|c|}{ Combustibles } & \multirow[b]{2}{*}{ Total } \\
\hline & & $\begin{array}{c}\text { Gaz } \\
\text { butane }\end{array}$ & $\begin{array}{c}\text { Gaz } \\
\text { /charbon }\end{array}$ & charbon & $\begin{array}{c}\text { Gaz } \\
\text { /bois/ } \\
\text { charbon }\end{array}$ & \\
\hline \multirow[t]{3}{*}{$\begin{array}{l}\text { Fréquence } \\
\text { crises }\end{array}$} & $\begin{array}{l}\text { Pas de crise } \\
\text { depuis plus } \\
\text { d'un an }\end{array}$ & $81 \%$ & $13 \%$ & $6 \%$ & $00 \%$ & $\begin{array}{c}100 \\
\%\end{array}$ \\
\hline & $\begin{array}{l}\text { Stade } 1 \text { (moins } \\
\text { d'une crise par } \\
\text { semaine) }\end{array}$ & $41 \%$ & $52 \%$ & $5 \%$ & $2 \%$ & $\begin{array}{c}100 \\
\%\end{array}$ \\
\hline & $\begin{array}{c}\text { Stade } 2 \\
\text { (environ } 3 \\
\text { crises par } \\
\text { semaine) }\end{array}$ & $7 \%$ & $87 \%$ & $2 \%$ & $4 \%$ & $\begin{array}{c}100 \\
\%\end{array}$ \\
\hline
\end{tabular}

Les données présentées dans ce tableau sont relatives aux fréquences de crises en fonction du type de combustibles utilisés dans le ménage. Il ressort que pour les 16 asthmatiques qui ne font plus de crises depuis plus d'un an, $81 \%$ soit 13 asthmatiques utilisent exclusivement le gaz. Contrairement à eux, la majorité des asthmatiques se trouvant au stade 1 ou au stade 2 de leur maladie et qui combinent les combustibles gaz et charbon ont une proportion respective de $52 \%$ pour 53 asthmatiques et de $87 \%$ pour 71 asthmatiques.

Les asthmatiques ne faisant plus de crises depuis plus d'un an optent pour des actions susceptibles de favoriser une amélioration de leur état de santé comme en témoigne l'analyse du discours de certains :

« Je trouve que le gaz est propre et selon les conseils que j'ai reçus, il dégage moins de pollution. Il est mieux de choisir ce combustible quand on a cette maladie ». (Homme, 53 ans, Nouveau quartier)

" Avant, j'utilisais le charbon de temps en temps, quand mon fils a été déclaré asthmatique à l'âge de 11 ans et que nous avons commencé à consulter le médecin, c'est sur ces conseils même que j'ai arrêté. Notre espace n'est pas grand, donc le jour où j'utilise le charbon, la cendre s'éparpille trop et c'est possible qu'il puisse en respirer et créer des soucis ». (Femme, 40 ans, Toit Rouge)

Les $52 \%$ se trouvant au stade 1 ont une maladie contrôlée, ce qui signifie que la fréquence des crises est inférieure à une crise par semaine. Leur état de santé est stable. Par contre, les $86 \%$ du stade 2 ont un asthme non encore contrôlé, ils font 3 crises par semaine. Leur état de santé n'est pas stabilisé et les crises perturbent leurs différentes activités ; créant ainsi des 
absentéismes au travail ou à l'école. Et cela se perçoit dans les propos de cet élève du secondaire :

« J'ai dû manquer des cours par moment à cause des crises, et cela joue parfois sur mes résultats scolaires car ce n'est pas tous les enseignants qui sont compréhensifs ». (Jeune homme, 21 ans, Sideci)

\section{Choix thérapeutiques des asthmatiques}

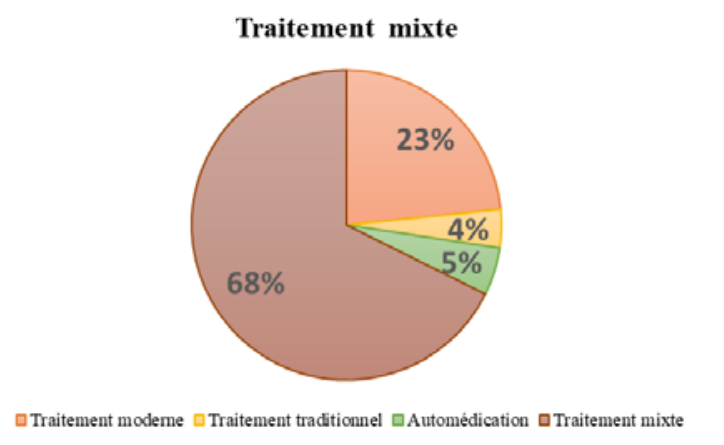

Figure 5. Choix thérapeutique des malades d'asthme de février à juillet 2018

La figure 5 indique que $68 \%$ des asthmatiques optent pour le traitement mixte (association du traitement moderne et du traitement traditionnel) tandis que $21 \%$ s'orientent uniquement vers le traitement. Le traitement traditionnel constitue le recours exclusif pour $4 \%$ des asthmatiques quand l'automédication est l'unique choix de 5\% d'entre eux.

La majorité des asthmatiques soit $68 \%$ a recours au traitement mixte du fait de la méconnaissance de la maladie ou la recherche désespérée de la guérison. En effet, l'ignorance ou la non acceptation des asthmatiques du caractère chronique ou incurable de la maladie les poussent à frapper à toutes les portes dans l'espoir de trouver la panacée. Cet état de fait se traduit dans les interviews des patients asthmatiques :

«Bon......quand la maladie a commencé c'était bizarre parce que personne ne connaissait cette maladie dans mon entourage. On ne savait pas comment soigner ça, donc je suis allé à l'hôpital, c'est là-bas qu'ils m'ont dit que c'est l'asthme. Mais, moi je pensais que j'allais être guéri et j'étais un peu déçu. J'ai alors commencé à plus me renseigner et faire des traitements traditionnels. C'est comme ça que je me suis retrouvé à faire le mélange des médicaments ». (Homme, 62 ans, Kouté)

"Il faut dire qu'au tout début, je soignais mon fils avec les médicaments prescrits uniquement par le médecin. Mais au bout d'un moment j'ai commencé à désespérer parce que son état ne s’améliorait pas, alors quand dans mon entourage on me parlait d'un médicament pour l'asthme, je me précipitais, je l'ai même envoyé chez un tradipraticien dans 
une ville de l'intérieur une fois. Malgré tout ça, son état ne s'est vraiment pas amélioré. Il fait trop de crises et souvent même il s'absente aux cours. Mais, finalement nous sommes revenus aux médicaments du médecin et vraiment je suis à la lettre ses recommandations et je crois qu'il y a de l'amélioration depuis un moment ». (Femme, 41 ans, Sideci)

À travers ces propos, nous percevons que la première orientation de la majorité des asthmatiques est l'hôpital. Mais, ils voient l'asthme comme une maladie curable après avoir pris les médicaments prescrits par le médecin. Ils se retrouvent très vite face à la réalité (la chronicité de la maladie). Les asthmatiques commencent dès lors à prendre toutes sortes de médicaments et à ne plus respecter les prescriptions du médecin. Ce mélange de médicaments aggrave pour la plupart leur état de santé par la répétition des crises. Parmi les 136 soit $68 \%$ d'asthmatiques qui utilisent le traitement mixte, nous allons rechercher le traitement prioritaire.

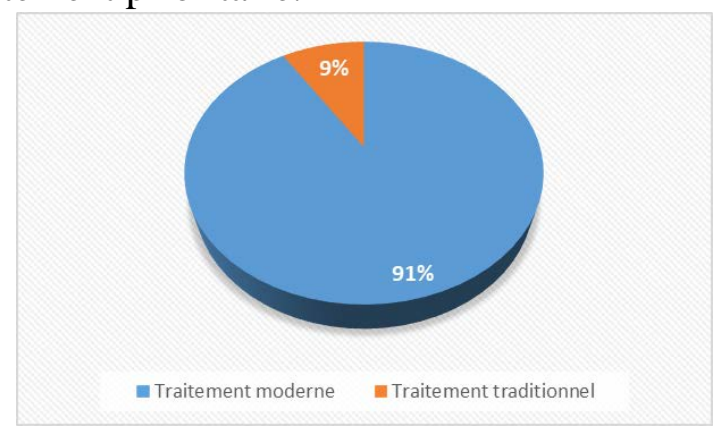

Figure 6. Répartition des enquêtés selon le choix du traitement (moderne ou traditionnel) de février à juillet 2018

L'étude du choix thérapeutique des asthmatiques a révélé que 136 asthmatiques soit $68 \%$ optaient pour le traitement mixte. En relevant leur priorité dans ce traitement, nous constatons que le traitement moderne est prioritaire avec un score de 91\% pour 123 asthmatiques. Le traitement traditionnel n'est prioritaire que pour 13 asthmatiques soit 9\%. La majorité des asthmatiques dans ce groupe qui a opté pour le traitement mixte a pour priorité le traitement moderne.

\section{Discussion}

Les résultats de l'étude montrent que les choix et habitudes des personnes asthmatiques concourent aussi bien à une amélioration qu'à une dégradation de leur état de santé. Il s'agit de la localisation du cadre de vie, du type de combustible utilisé dans le ménage et le choix thérapeutique adopté. Ces éléments, pour certains, sont sources de pollution de l'air intérieur. Ce résultat est corroboré par Kouao et al., (2019), qui dans leur étude effectuée dans les ménages à Lubafrique et à Andokoi dans la commune de 
Yopougon, ont montré après des mesures de la qualité de l'air intérieur/extérieur que le niveau de pollution en $\mathrm{PM}^{2,5}$ était au-dessus de la norme recommandée par l'OMS $\left(25 \mu \mathrm{g} / \mathrm{m}^{3}\right)$. C'est un constat qui s'explique par l'utilisation de la biomasse pour la cuisson des repas et l'insuffisance ou l'inadaptation du système d'aération (Kouao et al., 2019).

Selon l'OMS, la pollution de source ménagère est responsable de 7,7\% de la mortalité mondiale, et les combustibles utilisés y jouent un rôle (OMS, 2016). L'observation et l'analyse des données (45\% d'utilisateurs du charbon uniquement contre 55\% d'utilisateurs exclusifs du gaz butane) de la figure 3 donnent à croire que l'utilisation du gaz butane comme seule source de combustible représente un luxe pour la population. Nous en voulons pour preuves les déclarations suivantes de certains enquêtés :

« Hé! Je fais doucement, doucement avec ma bouteille de gaz là. C’est pourquoi j'utilise plus le charbon, parce que quand ça fini j'ai du mal à réunir l'argent pour recharger » (femme, 29 ans, Toit Rouge)

" J'utilise le gaz seulement pour des choses qui ne vont pas durer au feu. Sinon c'est charbon que j'utilise plus, c'est moins cher. Gaz là c'est bon mais souvent il n'y a pas l'argent pour recharger. C'est ce qui me fatigue » (femme, 35 ans, Lokoa)

" Charbon là même n'arrange pas oh, c'est salissant, c'est fatiguant. Mais je fais avec ce que j'ai, en espérant avoir mieux demain ; je compte le remplacer totalement par le gaz, mais pour le moment, je fais avec ce que j’ai, un peu un peu ça va aller » (femme, 31 ans, Bouet 2)

Le manque de moyens financiers contraint ces asthmatiques à utiliser le charbon qui, selon eux, est à moindre coût. Dans ces propos : «j'ai du mal à réunir l'argent pour recharger la bouteille » ou «c'est bon mais, souvent il n'y a pas l'argent pour recharger », "je fais avec ça », nous percevons que le choix d'utiliser plus le charbon n'est pas opéré de gaité de cœur par les enquêtés, mais plutôt pour des raisons économiques. Pour ces enquêtés, l'utilisation du gaz butane est réservée aux personnes ayant une meilleure situation socioéconomique. Dans ce contexte, les directives données par le médecin dans la gestion du cadre de vie pour une meilleure qualité de l'air intérieur ne sont pas suffisamment respectées.

Le taux de couverture en utilisation de gaz butane comme seul combustible est bas (30\%) dans la présente étude. Ce résultat va dans le même sens que l'étude de Sustainable Energy for all, où la couverture en utilisation de gaz butane est de $20 \%$ au niveau de la population ivoirienne (Sustainable Energy for all, 2012). L'utilisation du gaz butane comme seule source de combustible n'est pas suffisamment représentée en Côte d'Ivoire.

Pourtant, différentes pratiques quotidiennes permettent d'améliorer la qualité de l'air dans les domiciles. Il s'agit entre autres de choisir le combustible le moins polluant qui est le gaz butane comme le pratiquent les 
$30 \%$ des enquêtés, d'opter pour des méthodes moins polluantes dans le nettoyage et l'entretien de la maison et surtout de mettre l'accent sur l'aération du logement (bonne aération de toutes les pièces). L'aération concerne l'ouverture des portes et fenêtres pour le changement d'air dans la maison. L'amélioration de la qualité de l'air passe par un renouvellement d'air grâce à un système d'aération adapté à la taille du logement. C'est un geste simple et recommandé qui est à la portée de tous dans la lutte contre la pollution de l'air intérieur. Un logement mal aéré condense les polluants et représente un risque pour la santé. L'aération est perçue par les enquêtés comme une activité de routine qui est effectuée lors de l'entretien de la maison. Ce point de vue est partagé par Minoustchin et al., (2010) qui soutiennent que l'aération est généralement une pratique liée à une « habitude routinière » ou à une activité domestique « secondaire » insérée dans le cours d'une activité domestique « primaire » (faire le ménage, faire la cuisine, etc.).

Les individus ne perçoivent pas l'aération comme une forme de changement d'air, une manière d'améliorer la qualité de l'air dans leur domicile mais plutôt comme une sorte de culture, d'habitude acquise avec le temps. C'est un résultat est en congruence avec ceux de l'étude de Rosine et al., (2021). Dans cette étude, les représentations sociales de la pollution de l'air intérieur des asthmatiques étaient axées sur des éléments externes tels la poussière et la fumée qui pénètrent dans leur domicile. Les personnes asthmatiques trouvaient que maintenir les fenêtres fermées n'impactait pas la qualité de l'air dans le domicile étant donné que la pollution vient de l'extérieur. L'environnement dans lequel se situe la maison est un élément important. Certains enquêtés se plaignaient de la circulation de véhicules sur des rues non bitumées à proximité de leur domicile, de dépotoirs sauvages qui dégageaient des odeurs nauséabondes, du brulage de déchets ou pneus qui entrainaient la fumée dans leur maison. Toutes ces activités et situations contribuent à la dégradation de la qualité de l'air dans le logement. Selon Salameh et al, le risque d'être asthmatique ou d'avoir des symptômes respiratoires est non seulement lié à la pollution intérieure mais aussi extérieure, principalement le trafic routier. La probabilité de l'asthme était plus élevée chez les résidents près d'un fort trafic routier (ORa = 4,30 [IC95\% 1,45-12,71], $\mathrm{p}<0,05$ (Salameh et al, 2015).

L'asthme peut être contrôlé au moyen de médicaments et par l'application d'un certain nombre de mesures préventives. Il est donc important de pouvoir dépister cette maladie le plus rapidement possible, pour une prise en charge efficace qui permette d'éviter une aggravation. En termes de moyens préventifs, les combustibles utilisés jouent un rôle capital dans l'amélioration ou l'aggravation de l'état de santé de la personne asthmatique comme nous pouvons le constater dans le croisement des variables fréquence de crise et combustibles utilisés dans le ménage. De plus, le suivi et le respect 
des rendez-vous sont primordiaux dans la recherche d'un bon état de santé du malade. Dans ce contexte, quel choix thérapeutique adoptent les asthmatiques?

Concernant les choix thérapeutiques, notons qu'en Afrique la diversité des offres thérapeutiques est plus que jamais un fait avéré (Nkoma, 2015). Ce fait est relativement lié à l'existence de différentes pratiques et de croyances au sujet de la santé et de la maladie. Les malades adaptent leur parcours thérapeutique en priorité à leurs moyens financiers, à leur accès géographique aux formations sanitaires, et aux représentations sociales qu'ils se font de la maladie. Pour 68\% des asthmatiques, le traitement mixte (moderne et traditionnel) est le choix prioritaire dans la quête désespérée d’une guérison. Dans ces conditions, ils ne peuvent pas suivre correctement les prescriptions du médecin et perdent ainsi le contrôle sur la maladie. Contrairement au choix du traitement mixte chez les asthmatiques dans cette étude, Adjet et al. (2017) ont montré que la médecine traditionnelle était le choix privilégié chez les parents, dans le cadre des maladies infantiles dans la ville de Daloa. Ces parents justifient leur choix par une inefficacité de la médecine occidentale. En plus, ils trouvent que la médecine traditionnelle est accessible et à moindre coût.

Dans la présente étude, le choix du traitement mixte peut s'expliquer par la non-compréhension du patient de la nature chronique de l'asthme. Il cherche à tout prix à en être guéri définitivement. Certains ont la phobie de la prise de médicaments au quotidien ou sont inquiets des conséquences de ces médicaments sur leur organisme.

Ces différentes situations présentées entrainent une insuffisance du contrôle de la maladie. Les résultats ont montré que $41 \%$ des enquêtés étaient au stade 2 de la maladie faisant plus ou moins trois crises par semaine. C'est une proportion non négligeable qui a besoin d'informations et de formations sur la gestion et la maitrise de la maladie.

Le suivi du traitement est nécessaire dans la lutte contre cette maladie. Toutefois, il est aussi important d'éviter tout ce qui peut déclencher des crises, c'est-à-dire des stimulations provoquant des irritations ou des inflammations des voies respiratoires. Avec l'aide du médecin, chaque asthmatique doit maitriser son environnement et apprendre à éviter ce qui déclenche les crises. C'est une démarche préventive car elle consiste à éviter toutes les conditions favorables au déclenchement de la crise.

\section{Conclusion}

L'utilisation combinée du charbon de bois/gaz butane (63\%) des enquêtés et le recours au traitement mixte (traditionnel/ moderne) pour 68\% des enquêtés sont des choix raisonnés effectués par les asthmatiques. Ces raisonnements sont soutenus par le déficit de moyens financiers, la phobie 
d'être toujours sous traitement médicamenteux et le désir de guérir définitivement de la maladie. Cette multitude de choix thérapeutiques n'est aucunement bénéfique pour leur état de santé. A titre d’illustration, 41\% d'entre eux ont un asthme persistant léger, ils font environ trois crises par semaine. Il y a nécessité d'informer et d'éduquer ces personnes asthmatiques dans la prise en charge de leur maladie.

\section{Remerciements}

Cette étude a été soutenue par la Chaire Ecosanté « Pollution urbaine de l'air, Pollution de l'air intérieur et maladies non transmissibles » (Chaire Pol) ainsi que par le Centre de Recherche en Développement International (CRDI) à travers la subvention $\mathrm{N}^{\circ} 107347-001$.

\section{References:}

1. OMS (2017). Global, regional, and national incidence, prevalence, and years lived with disability for 328 diseases and injuries for 195 countries, 1990-2016: A systematic analysis for the Global Burden of Disease Study 2016 . Lancet 2017; 390: 1211-59

2. Puddu, M. ; Bayingana, K., et Tafforeau, J., (2003). L'Asthme et la Pollution de l'air. Rapport. Bruxelles (Belgique). Repéré à http://infoairinterieur.fr/wp-content/uploads/2016/01/169.pdf

3. 3. Billionnet, C., (2012). Pollution de l'air intérieur et santé respiratoire: prise en compte de la multi-pollution. Santé publique et épidémiologie. (Thèse de Doctorat). Université Pierre et Marie Curie Paris VI. https://tel.archives-ouvertes.fr/tel-00827664

4. Nicolas, M. ; Chiappini, L., et D’Anna, B., (2013). Activités domestiques et qualité de l'air intérieur: émissions, réactivité et produits secondaires. Rapport. Programme de recherche interorganisme pour une meilleure qualité de l'air, France, 149p

5. Lang, K. (2006). Indoor Air Pollution (Pollution de l'air intérieur)-Etat de connaissances et situation au Sénégal. PERACOD / FASEN, Rapport de fin de stage.

6. Dallongeville, A., (2015). Exposition cumulée aux contaminants de l'air intérieur susceptibles d'induire des affections respiratoires chroniques de l'enfant. (Thèse de Doctorat) Rennes 1. http :www.theses.fr/2015REN1B013 \#

7. Liu, Q. ; Wang, W. et Jing, W., (2018). Indoor air pollution aggravates asthma in Chinese children and induces the changes in serum level of miR-155, International Journal of Environmental Health Research, 29(1), 22-30. DOI: 10.1080/09603123.2018.1506569

8. Koné, A. ; Koffi , M.O.B. ; Djegbeton, E. ; Ahui, B.J.M. ; BrouGode, V.C. ; Ngom, A.; Horo, K. ; Kouassi, B.A. ; Koffi, N. ; Aka- 
Danguy, E., (2018). Epidémiologie de l'asthme de l'adolescent en milieu scolaire de la ville de Bouaké. https://doi.org/10.1016/j.rmr.2018.10.111

9. Koné, A. ; Koffi , M.O.B. ; Horo, K. ; Djegbeton, E. ; Ahui, B.J.M. ; Brou-Gode, V.C.; Ngom, A.; Kouassi, B.A. ; Koffi, N. ; AkaDanguy, E., (2018). Epidémiologie de l'asthme de l'adolescent en milieu scolaire de la ville de Korhogo. https://doi.org/10.1016/j.rmr.2018.10.179

10. Bozonnet, J.P. (2007) : Conscience écologique et pratiques environnementales, Pacte-Institut d'Etude Politique, Grenable, pp 279-287.

11. INS, 2014. Côte d'Ivoire : Les résultats du recensement de 2014. Repéré à https://atlasocio.com/revue/demographie/2015/cote-divoire-les-resultats-du-recensement-de-2014.php. Le 06/06/2021

12. Yopougon info. (2014). La commune de Yopougon. En ligne http://yopougon-info.blogspot.com/2014/09/presentation-de-lacommune.html. Consulté le 03/12/2018

13. Abric, J.-C., (2005). Introduction. In J.-C. Abric, Méthodes d'étude des représentations sociales, ERES « Hors collection », 7-10.DOI 10.3917/eres.abric.2003.01.0007, pp 7-10

14. N’Da, P., (2002). Méthodologie de la recherche: de la problématique à la discussion des résultats, Abidjan, EDUCI.

15. GINA. (2019). Guide de poche destiné aux professionnels de santé, Révisé en 2019. Repéré à :

https://ginasthma.org/wp-content/uploads/2019/09/GINA-2019-mainPocket-Guide-French-wms.pdf

16. Kouao, A.K.R.; N'datchoh, E.T.; Yoboue, V.; Silue, S.; Attoh, H.; Coulibaly, M.; Robins, T., (2019). Exposure to indoor and outdoor air pollution among children under five years old in urban area. Global J. Environ. Sci. Manage., 5(2): *_*.

DOI: 10.22034/gjesm.2019.02.00*url:http://gjesm.net/***

17. OMS. (2016). Preventing disease through healthy environments A global assessment of the burden of disease from environmental risks. Organisation Mondiale de la Santé. Genève.

http://www.who.int/indoorair/

18. Sustainable Energy for all, 2012. Evaluation rapide et analyse des GAPS de la Côte d'Ivoire. Repéré à

https://webcache.googleusercontent.com/search?q=cache:e6BRbfvB_

8J:https://www.seforall.org/sites/default/files/l/2015/05/Cote_dIvoir e_RAGA.pdf+\&cd=1\&hl=fr\&ct=clnk\&gl=ci. Le 27/05/2021

19. Minoustchin, M.; et Vera-Navas, G. (2010). Représentations et comportements de gestion de la qualité de l'air intérieur dans les 
logements. Pollution atmosphérique [En ligne], $\mathrm{N}^{\circ} 206$, mis à jour le:07/09/2015.http://lodel.irevues.inist.fr/pollutionatmospherique/index.php?id=678

20. Ahou Rosine K., Fulbert T. et Soualiho O. (2021). Représentations sociales de la pollution de l'air intérieur et pratiques sociales des malades d'asthme de la commune de Yopougon (Côte d'Ivoire). European Scientific Journal, ESJ, 17(14), 217. https://doi.org/10.19044/esj.2021.v17n14p217

21. Salameh, P., Karaki, C., Awada, S., Rachidi, S., Al Hajje, A., Bawab, W., . . . Waked, M. (2015). Asthme, pollutions intérieure et extérieure : étude pilote chez des adolescents libanais scolarisés. Revue des Maladies Respiratoires, 32(7), 692-704. doi: http://dx.doi.org/10.1016/j.rmr.2014.11.073

22. Nkoma, P.P. (2015). Itinéraires thérapeutiques des malades au Cameroun : Les déterminants du recours à l'automédication. 7ème Conférence sur la Population Africaine: "Dividende Démographique en Afrique: Perspectives, Opportunités et Défis”. Johannesburg, Afrique du Sud., <http://uaps2015.princeton.edu/>. < hal-0133941

23. Adjet A., Zadou Z., et Mafou K. (2017). Pratiques Thérapeutiques Locales et Maladies Infantiles Dans le District Sanitaire de Daloa (Centre - Ouest Côte d'Ivoire). European Journal of Social Sciences ISSN 1450-2267, 55( 3), pp.286-296 